\title{
Parallel phase-shifting self-interference digital holography with faithful reconstruction using compressive sensing
}

\author{
Yuhong Wan, ${ }^{1,2,{ }^{*}}$ Tianlong Man, ${ }^{1,2}$ FAn Wu, ${ }^{1,2}$ MyUng K. KIM, ${ }^{3}$ And Dayong \\ WANG $^{1,2}$
}

${ }^{1}$ College of Applied Sciences, Beijing University of Technology, 100 Ping Le Yuan, Chaoyang District, Beijing 100124, China

${ }^{2}$ Beijing Engineering Research Centre of Precision Measurement Technology and Instruments, Beijing University of Technology, 100 Ping Le Yuan, Chaoyang District, Beijing 100124, China

${ }^{3}$ Department of Physics, University of South Florida, Tampa, Florida 33620, USA

*Corresponding author: yhongw@bjut.edu.cn

We present a new self-interference digital holographic approach that allows single-shot capturing threedimensional intensity distribution of the spatially incoherent objects. The Fresnel incoherent correlation holographic microscopy is combined with parallel phaseshifting technique to instantaneously obtain spatially multiplexed phase-shifting holograms. The compressivesensing-based reconstruction algorithm is implemented to reconstruct the original object from the under sampled demultiplexed holograms. The scheme is verified with simulations. The validity of the proposed method is experimentally demonstrated in an indirectly way by simulating the use of specific parallel phaseshifting recording device.

Key Words: Optical Metrology, Holography; Digital holography; Phase shift; Compressive sensing, Image reconstruction techniques.

\section{Introduction}

Three-dimensional (3D) microscopic imaging techniques have been significant tools for discovering the mechanisms involved in biological cells and tissues [1]. Among them, digital holography has been used for various applications because it offers the advantages of scanning-free, simplicity, and potential to track rapidly moving samples in volume [26]. However, the validity of the conventional digital holographic techniques is challenged while one want holographic imaging for spatially incoherent objects. To solve the problem, self-interference digital holography (SIDH) has been proposed and developed [7]. Holographic recording is implemented by exploring the spatial selfcoherence property of the point object in SIDH. The performances of such kind of self-interference digital holographic systems have been investigated under different optical setups [8-10], and further used for aberration correction [11,12], confocal microscopy [13] and color imaging $[14,15]$. The high-resolution 3D SIDH imaging systems great benefit the biological investigation, and provide a high-efficiency tool for the 3D tracking of the fluorescent particles. Phase-shifting (PS) technique has been implemented to eliminate the twin image and zero order in digital holography. However, temporal resolution of the system is usually reduced because three or more holograms have to be recorded sequentially. Thus the imaging of rapidly moving object is of great challenge. Although off-axis hologram can be recorded using particular optical setups, the signal to noise ratio of the reconstructed image is relatively low [15], or the field of view of the system is reduced
[16]. On the other hand, parallel phase-shifting (PPS) digital holography is capable of instantaneous measurement by capturing several spatially multiplexed holograms with single-shot exposure [1719]. However, the quality of the reconstructed image is reduced because the demultiplexed holograms are under-sampled. Fortunately, by designing the holographic recording and reconstruction processes using compressive sensing (CS), the under-sampled signals can be accurately inferred $[20,21]$.

We propose a parallel phase-shifting self-interference digital holography (PPSSIDH) which is capable of instantaneous capturing and reconstructing the three-dimensional intensity distribution of spatially incoherent object. The holographic recording in the proposed method as a CS scheme and the reconstrusction of the hologram as an inverse problem are demonstrated. The CS reconstruction allows us to accurately rebuild signals at a sampling rate much lower than Shannon's limit by exploiting sparsity. In this paper, four phase-shifted holograms are spatially multiplexed in a single-shot captured PPS hologram. The PPS hologram are then demultiplexed into four undersampled phase-shifted holograms with sampling rate of only $25 \%$. Combing with CS reconstruction, noise introduced by the undersampling is suppressed by exploring the sparsity of the object under CS frame work.

\section{Methodology}

The schematic diagram of the proposed method is shown in Fig. 1. The point source located at $f_{0}-z_{s}$ from the Lens (with focal length of $f_{0}$ ) emits a spherical wave. The roughly collimated beam illuminates the spatial light modulator (SLM) and is separated into two beams after reflected by the SLM. The SLM is served as a diffractive optical element (DOE) by uploaded a suitable mask. Assume the mask is designed that randomly half of the pixels are used to display the phase of a positive lenses with focal lengths of $f_{a}$ and the remaining pixels are used to display the phase of a positive lenses with focal lengths of $f_{b \text { : }}$ Thus the two beams after SLM are converged to point $a$ located at $f_{1}$ from SLM and point $b$ located at $f_{2}$ from SLM, respectively. The two beams can interfere with each other because they originated from the same point The interference pattern is coined as point source hologram (PSH) here. The intensity distribution of PSH is similar to Fresnel zone plate, where the 3D spatial coordinates of the corresponding point source is encoded.

For general case, the hologram of an extended object is the two dimensional convolution of the intensity distribution of the object and the PSH. At the distance of $z_{h}$ from SLM, an image sensor with a PPS 
filter consisting of an array of $2 \times 2$ elements is used to capture the hologram. For each element, transmittance of the 4 pixels are designed in such a way that four different PS values of $0,-\pi / 2,-\pi$, and $-3 \pi / 2$ are generated between the two interference beams. The recorded hologram $h$ can be demultiplexed into four under-sampled phaseshifted holograms $h_{1}, h_{2}, h_{3}$, and $h_{4}$ using the demultiplexing masks $m_{1}$, $m_{2}, m_{3}$, and $m_{4}$ as shown in Fig. 1. The twin image and zero order can be eliminated, and the complex hologram $C$ can be obtained using the equation of [11]:

$$
\begin{aligned}
C & =\left(m_{1} \cdot h-m_{3} \cdot h\right)-j\left(m_{2} \cdot h-m_{4} \cdot h\right) \\
& =\left(h_{1}-h_{3}\right)-j\left(h_{2}-h_{4}\right) .
\end{aligned}
$$

The 3D object data $O(x, y, z)$ can be reconstructed from the complex hologram by calculating the Fresnel propagation formula based on angular spectrum method as:

$$
O(x, y, z)=\mathcal{F}^{-1}\left\{\mathcal{F}(C) \cdot \exp \left[-j \pi \lambda z_{r}\left(u^{2}+v^{2}\right)\right]\right\},
$$

where $\mathcal{F}$ and $\mathcal{F}^{1}$ denote Fourier and inverse Fourier transform, respectively; $u$ and $v$ are the spatial frequency coordinates, $z_{r}$ is reconstruction distance. The quality of the reconstruction $O(x, y, z)$ is reduced because the four phase-shifted holograms are all undersampled. To solve the problem, a generalized framework to acquire multichannel optical data using CS has been proposed and numerically investigated in Fresnel holography [21]. The successfully implementation of compressive sensing relies on two requirements: Sparsity and coherence of the sensing mechanism. Here the sparsity is implemented by expressing the object in some domain such as total variation (TV) and discrete Fourier transform (DFT). The second requirement "coherence" is quite different with the concept of "coherence" in the statistical optics. It means that the sensing operation and sparsifyication operation should be mutual incoherent. From another view, the coherence also can understand that the object information (such as, the diffraction pattern of the object) should be evenly spread over the set of basis that describe it at the recording plane. By exploring the spatial-self-coherence of the point object, 3D information of the spatially incoherent object can be encoded into the Fresnel hologram using SIDH system. Hence as commonly done in other compressive digital holographic applications [20, 21, 23, 24, 26], Fresnel diffraction is utilized as sensing operation in the paper. The CS reconstruction method is implemented to infer the reconstructions from the under-sampled phase-shifted holograms and improve the imaging quality in PPSSIDH. In practice, four reconstructions are inferred from the four under-sampled phase-shifted holograms using CS algorithm respectively, then the reconstructions are combined to suppress the twin image and zero order. The observation process of the system for each under-sampled hologram can be expressed with linear algebra as:

$$
h_{i}=\left.H_{i} f_{i}\right|_{i=1,2,3,4},
$$

where $h_{i}$ is the vector under-sampled hologram, $f_{i}$ is the vector reconstruction corresponding to each under-sampled phase-shifted hologram, and

$$
H_{i}=\left.m_{i} \mathcal{F}^{-1} Q \mathcal{F}\right|_{i=1,2,3,4}
$$

is the operator where $m_{i}$ is the vector demultiplexing mask and $Q$ is the vector propagation operator. The system model as shown in Eq. (3) is linear and ill-posed [20]. We use a specific CS algorithm, two-step iterative shrinkage/thresholding (TwIST) [22], to infer the reconstructions by solving the following optimization:

$$
\hat{f_{i}}=\underset{f_{i}}{\arg \min }\left\|h_{i}-H_{i} f_{i}\right\|_{l 2}+\left.\alpha\left\|f_{i}\right\|_{T V}\right|_{i=1,2,3,4},
$$

where $\|\cdot\|_{12}$ indicates the Euclideam norm, $\alpha$ is regularization parameter and $\|\cdot\|_{T V}$ indicates the two-dimensional total variation (TV) regularizer. Finally, the reconstructed object data without twin image and zero order is obtained by combining the resulting four reconstructions. Rather than the random sampling method, the structured subsampling as done by the using of parallel phase shifting-mask is also valid in CS reconstruction [23]. The detailed procedure of the proposed CS reconstruction of PPSSIDH hologram is:

\section{Hologram is recorded using the digital camera with PPS filter array. The size of raw hologram is assumed to be $N_{x} \times N_{y}$ pixels;}

2. Four under-sampled phase-shifted holograms are extracted from the raw hologram. The extraction is implemented by multiplying the raw hologram with the four demultiplexing masks (as shown in Fig. 1) respectively. All the four extracted phase-shifted hologram are under-sampled, because for each of them the size is $N_{x} \times N_{y}$ pixels, but $75 \% \times N_{x} \times N_{y}$ of pixel values are missed (set to be zero);

3. Basing on the model of Eq. (3), the four under-sampled holograms are CS reconstructed respectively using Eq. (5). The size of each resulting reconstructions are still $N_{x} \times N_{y}$ pixels, but all the missed pixel values are inferred accurately;

4. The four reconstructions in step 3 are combined following four-step phase shifting method (similar with the procedure in Eq. (1)). The twin image and zero order is suppressed, as well as the image degradation problem caused by the information missing during the reconstruction of under-sampled holograms is solved using CS methods.

\section{Results and discussion}

The validity of the proposed method was verified with simulations. The self-interference digital holographic recording procedure as shown in Fig. 1 was simulated with the parameters of: $f_{0}=250 \mathrm{~mm}$ and central wavelength of the light was $\lambda=650 \mathrm{~nm}$. For simplicity, the pixel pitch of SLM were assumed to be the same $(\Delta=19 \mu \mathrm{m})$ as image sensor, and their pixel numbers are both $N_{x} \times N_{y}=512 \times 512$ pixels. One of the focal length of the DOE on SLM was $f_{a}=300 \mathrm{~mm}$. The recording distance was chosen as $z_{h}=500 \mathrm{~mm}$, which is larger than $N_{x} \Delta^{2} / \lambda=142 \mathrm{~mm}$ to achieve maximum performance of CS algorithm [24]. The other focal length of the DOE was chosen as $f_{b}=z_{h} f_{d} /\left(2 f_{a}-z_{h}\right)=1500 \mathrm{~mm}$ according with the condition in [25] to optimize the numerical aperture of the hologram and hence improves the imaging quality of the system. The object was located at the front focal plane of the Lens, thus $z_{s}=0, f_{1}=f_{a}$ and $f_{2}=f_{b}$. The reconstruction distance can be calculated as $z_{r}=\left\{\left(f_{1}-z_{h}\right)\left(f_{2-}\right.\right.$ $\left.\left.\left.z_{h}\right) /\left[\left(f_{2}-z_{h}\right)-\left(f_{1}-z_{h}\right)\right]\right\}\right\}$. Angular spectrum method is used during the conventional reconstruction of the holograms, while the main advantage is that the pixel size is kept invariant during the propagation. Filter window is adopted to solve the restriction on propagation distance [27]. For a $19 \mu \mathrm{m}$ diameter point source object, the corresponding reconstructed intensity image obtained using propagation method as shown in Eq. (2), which can be considered as the point spread function (PSF) of the system, is shown in Fig. 2(a). Some noise around the central spot that introduced by the undersampled phase-shifted holograms is obvious. Using TwIST, reconstructions corresponding to each under-sampled hologram were obtained and then combined to get the result as shown in Fig. 2(b). The whole procedure takes 1020 s (500 iterations, Inter Core i7-2600 CPU @ 3.4Ghz, 3.24GB RAM). The noise introduced by the insufficient sampling of each hologram was suppressed. The improvement of the quality of PSF can be observed more clearly from the magnified results in Fig. 2(c) and Fig. 2(d). 
Then the method was applied to an extended object, the "Lena" image, while the other parameters were kept the same as those in the above simulation. Grid-like noise reduces the quality of the result obtained using propagation method as shown in Figs. 3(a) and 3(c). This noise was greatly suppressed in CS reconstructed results as shown in Figs. 3(b) and 3(d). The validity of the proposed method in improving the imaging quality was demonstrated.

Experiments were carried out using the optical setup as shown in Fig. 4. The resolution chart S (1951 USAF hi-resolution target, negative, Edmund Optics) was back-illuminated by a roughly collimated light beam from LED source $(\lambda=625 \mathrm{~nm}$, bandwidth $16 \mathrm{~nm}, \mathrm{M} 625 \mathrm{~L} 3$, Thorlabs). A diffuser D was used for spatially incoherent illumination.

An infinity-corrected microscope objective OBJ (Plan Fluor, 20×, $\mathrm{NA}=0.45$, Nikon) was used to image the sample. The back aperture of the OBJ was projected directly onto the tube lens L3 (with focal length of $\left.f_{3}=300 \mathrm{~mm}\right)$ by use of a 4 relay system consist of $\mathrm{L} 1\left(f_{1}=100 \mathrm{~mm}\right)$ and L2 $\left(f_{2}=100 \mathrm{~mm}\right)$. A polarizer $P$ was inserted and set at a $45^{\circ}$ angle to the

SLM $(1920 \times 1080$ pixels, $8 \mu \mathrm{m}$ pixel pitch, phase only modulation, PLUTO, Holoeye) sensitive axis (along horizontal). The DOE displayed on the SLM is equivalent to a positive lens with focal length of $f_{\mathrm{a}}=1070 \mathrm{~mm}$. After reflection by the SLM, the horizontal polarization component of the beam was modulated by the DOE, while the vertical polarization component was reflected by the SLM as if from a plane mirror. Therefore two images of the resolution chart appeared at the distances of $172 \mathrm{~mm}$ and $205 \mathrm{~mm}$ to the SLM, respectively. The CCD to SLM distance was set to be $187 \mathrm{~mm}$ to achieve the above mentioned imaging quality optimization condition. A quarter wave plate QWP set at $45^{\circ}$ angle was used to transform the beams into two circular waves with opposite polarization direction. Note that for the purpose of simultaneously recording phase-shifting holograms, a polarizer array with four different linear polarization directions similar as described in [17-19] can be attached before the image sensor chip of CCD camera. Though the cameras with such kind of polarizer array have already been commercially available, here are still some restrictions such as the price or fabrication problems that limit the applications. To prove the validity of the proposed method, here single-piece polarizer was used in front of the camera to simulate the using of the polarizer array. It should be emphasized that through the lack of using the polarizer array in this paper, the basic principle about the generation of four phase-shifting steps is the same with one used in parallel-phaseshifting.

In the experiment, four holograms were captured by the CCD (2048

$\times 2048$ pixels, $7.4 \mu \mathrm{m}$ pixel pitch, ML4022, FLI) while the polarizer were rotated corresponding to the four directions of the polarization array. The hologram is resized to $1000 \times 1000$ pixels. Then $25 \%$ of the pixels of each hologram were extracted using digital masks and combined to generate a simulated PPSSIDH single-shot captured hologram as shown in Fig. 5(a). Fig. 5(b) shows the reconstructed image obtained basing on propagation method. The object was not reconstructed because of the insufficient sampling, and the peak signal to noise ratio (PSNR) is 14.8dB. On the other hand, the object was well reconstructed as shown in Fig. 5(c) using CS method. The quality of the reconstruction was greatly improved as the noise introduced by the insufficient sampling was mostly suppressed, and the PSNR is $30.9 \mathrm{~dB}$. The whole procedure takes 3240s (400 iterations, Inter Core i7-2600 CPU @ 3.4Ghz, 3.24GB RAM).

In PPSSIDH, CS reconstruction algorithm was used to solve the image degradation problem caused by the insufficient sampling of demultiplexed phase-shifted holograms. The proposed method is tested under a self-interference holographic microscopic imaging system, and the objects are shown to be reconstructed accurately from four 25\% sampled phase-shifted holograms. However, in Fig. 5(c), we note that the element 3 of group 9 (with spatial frequency of 645 line pairs $/ \mathrm{mm}$, approaching the Rayleigh resolution limit of $0.61 \lambda / \mathrm{NA}=850 \mathrm{~nm}$ ) was not resolved because of the imperfect modulation characteristic and optical surface of the polarizer used in the experiment. This problem can be solved with improvement of polarization optical elements. In our existing method, the four holograms have to be CS reconstructed respectively, because in Fig. 1 and Eq. (4) the spatial distribution of four demultiplexing masks is different. For CS reconstruction of each under-sampled phase-shifted hologram, the twin image and zero order are not suppressed yet. Thus the effectiveness of the TV-based algorithm in finding the accurate solution is reduced and further degrades the quality of final result. To solve the problem, a synthetically CS reconstruction algorithm can be built, where TV regularization can be implemented directly on the final result, rather than on each under-sampled phase-shifted hologram separately.

\section{Conclusion}

In summary, we have proposed and proved the concept of a method for single-shot capturing of 3D information of spatially incoherent illuminated or self-luminous object using PPSSIDH. Four undersampled phase-shifted holograms are demultiplexed from a singleshot captured interference pattern recorded by an image sensor combining with polarizers. The object is faithfully reconstructed with a CS algorithm. The concept was verified by simulations and experiments with simulating use of the polarizer array. Through four holograms are recorded sequentially in the experiments, the concept of accuracy reconstruction the object information using CS has been proofed. The simultaneously capturing of the hologram can be achieved by simply adopt the polarizer array similar as described in [17-19]. So the proposed method has great potential to be applied in 3D tracking of spatially incoherent samples, such as the fluorescence particles or proteins in some biological researches. It should be noticed that, although Fresnel propagation is used as the sensing operator here, the detail expression of the sensing matrix is quite. Thus by further exploring the coherence parameter and comparing it with the conventional Compressive Fresnel holography, more potential advantages can be discovered. With the development of image sensors with the spatially multiplexed polarization and/or spectral filter, the proposed method in this letter has unique advantages in the 3D/multidimensional data acquisition and reconstruction applications. Future work will focus on the imaging resolution enhancement by investigating the CS reconstruction guarantees in self-interference digital holography.

Funding. National Science Foundation of China under Grant No. 61575009 and Grant No.61107002, the Scientific Research Project from Beijing Municipal Education Committee under Grant No.km201410005032, and the Project from Beijing Municipal Organization Department under No. Q0006111201401.

\section{References}

1. D. J. Stephens, and V. J. Allan, Light microscopy techniques for live cell imaging,"Science 300, 82 (2003).

2. M. Habaza, B. Gilboa, Y. Roichman, and N. T. Shaked, Tomographic phase microscopy with $180^{\circ}$ rotation of live cells in suspension by holographic optical tweezers,Opt. Lett. 40, 1881 (2015).

3. G. D. Caprio, A. E. Mallahi, P. Ferraro, R. Dale, Gianfranco Coppola, B. Dale, Giuseppe Coppola, and F. Dubois, 4D tracking of clinical seminal samples for quantitative characterization of motility parameters Biomed. Opt. Express 5, 690 (2014).

4. P. Ferraro, S. Grilli, D. Alfieri, S. De Nicola, A. Finizio, G. Pierattini, B. Javidi, G. Coppola, and V. Striano, Extended focused image in microscopy by digital holography, Opt. Express 13, 6738 (2005).

5. G. Di Caprio, P. Dardano, G. Coppola, S. Cabrini, and V. Mocella, Digital holographic microscopy characterization of superdirective beam by metamaterial, Opt. Lett. 37, 1142 (2012). 
6. G. G. Romero, A. C. Monaldi, and E. E. Alanis, Digital holographic microscopy for detection of Typanosoma cruziparasites in fresh blood mounts, Opt. Commun. 285, 1613 (2012).

7. J. Rosen, and G. Brooker, Non-scanning motionless fluorescence threedimensional holographic microscopy, Nat. Photon. 2, 190 (2008).

8. D. N. Naik, G. Pedrini, and W. Osten, Recording of incoherent-object hologram as complex spatial coherence function using Sagnac radial shearing interferometer and a Pockels cell, Opt. Express 21, 3990 (2014).

9. J. Rosen, N. Siege, and G. Brooker, Theoretical and experimental demonstration of resolution beyond the Rayleigh limit by FINCH fluorescence microscopic imaging, Opt. Express 19, 26249 (2011).

10. J. Rosen, and R. Kelner, Modified Lagrange invariants and their role in determination transverse and axial imaging resolutions of selfinterference incoherent holographic systems Opt. Express 22, 29048 (2014).

11. M. K. Kim, Adaptive optics by incoherent digital holography, Opt. Lett. 37, 2694 (2012).

12. M. K. Kim, Incoherent digital holographic adaptive optics, Appl. Opt. 52, A117 (2013).

13. N. Siegel, and G. Brooker, Improved axial resolution of FINCH fluorescence microscopy when combined with spinning disk confocal microscopy, Opt. Express 22, 22298 (2014).

14. M. K. Kim, Full color natural light holographic camera, Opt. Express 21, 9636 (2013).

15. Y. H. Wan, T. L. Man, and D. Y. Wang, Incoherent off-axis Fourier triangular color holography, Opt. Express 22, 8565 (2014).

16. R. Kelner, and J. Rosen, Spatially incoherent single channel digital Fourier holography, Opt. Lett. 37, 3723 (2012).
17. Y. Awatsuji, M. Sasada, and T. Kubota, Parallel quasi-phase-shifting digital holography, Appl. Phys. Lett. 85, 1069 (2004).

18. Y. Awatsuji, A. Fujii, T. Kubota, and O. Matoba, Parallel three-step phase-shifting digital holography, Appl. Opt. 45, 2995 (2006).

19. Y. Awatsuji, T. Tahara, A. Kaneko, T. Koyama, K. Nishio, S. Ura, T. Kubota, and O. Matoba, Parallel two-step phase-shifting digital holography, Appl. Opt. 47, D183 (2008).

20. D. J. Brady, K. Choi, D. L. Marks, R. Horisaki, and S. Lim, Compressive holography, Opt. Express 17, 13040 (2009).

21. R. Horisaki, J. Tanida, A. Stern, and B. Javidi, Multidimensional imaging using compressive Fresnel holography, Opt. Lett. 37, 2013 (2012).

22. J. M. Bioucas-Dias, and M. A. T. Figueiredo, A new twist: Two-step iterative shrinkage/thresholding algorithms for image restoration, IEEE Transactions on Image Processing 16, 2992 (2007).

23. Y. Rivenson, A. Stern, and B. Javidi, Overview of compressive sensing techniques applied in holography, Appl. Opt. 52, A423 (2013).

24. Y. Rivenson, and A. Stern, Conditions for practicing compressive Fresnel holography, Opt. Lett. 36, 3365 (2011).

25. Y. H. Wan, T. L. Man, H. Chen, Z. Q. Jiang, and D. Y. Wang, Effect of wavefront properties on numerical aperture of Fresnel hologram in incoherent holographic microscopy, Chin. Phy. Lett. 31, 044203-1 (2014).

26. P. Ramachandran, Z. C. Alex, and A. Nelleri, Compressive Fresnel digital holography using Fresnelet based sparse representation, Opt. Commun. 340, 110 (2015).

27. N. Siegel, J. Rosen, and G. Brooker, Faithful reconstruction of digital holograms captured by FINCH using a Hamming window function in the Fresnel propagation, Opt. Lett. 38(19), 3922 (2013). 

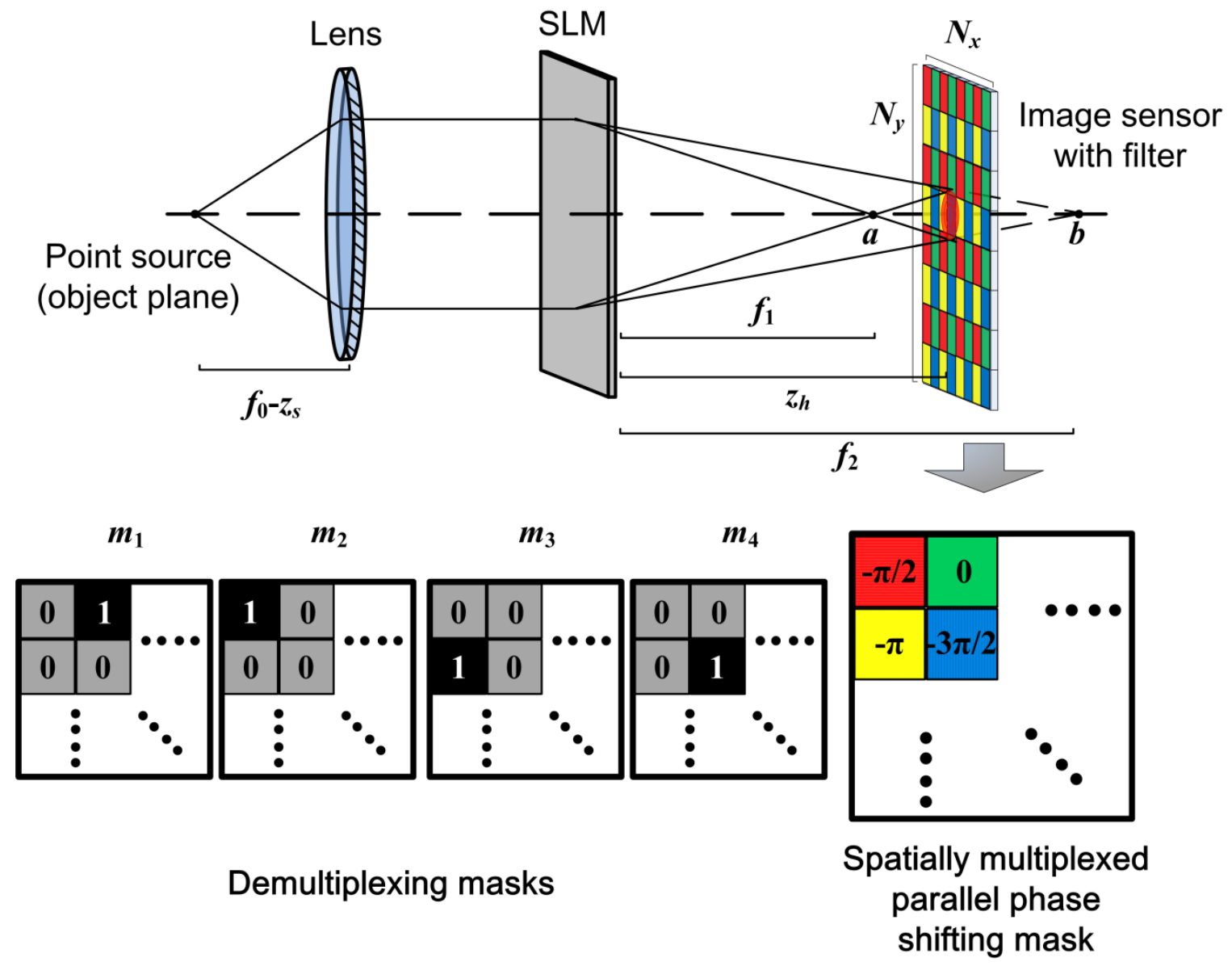

Fig. 1. Schematic of the proposed parallel phase-shifting self-interference digital holography system. 


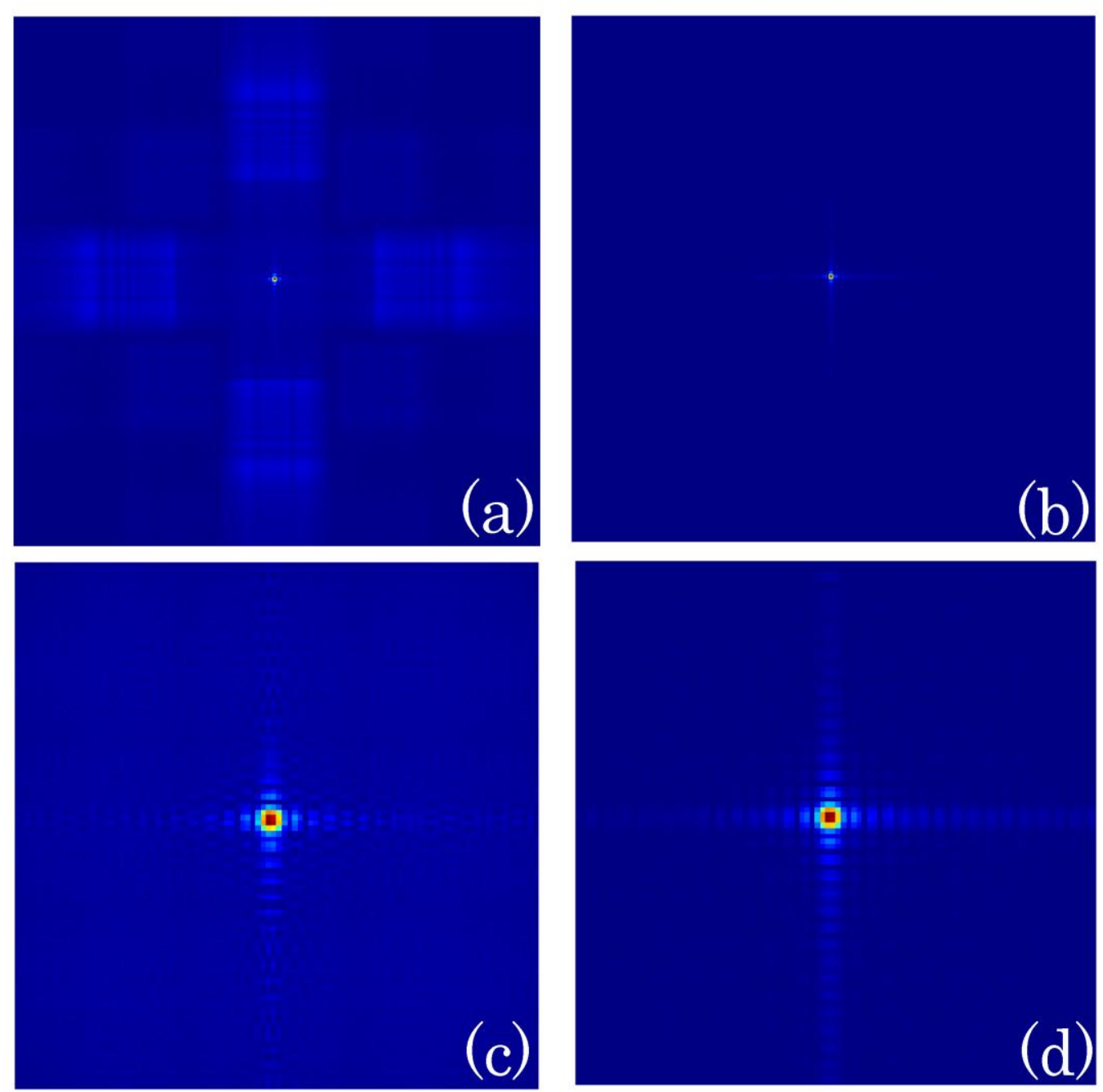

Fig. 2. Simulated reconstructed results of the proposed method. Reconstructions of a point object using (a) Propagation method and (b) CS algorithms, (c) and (d) show the corresponding magnified area from (a) and (b) at the image center.

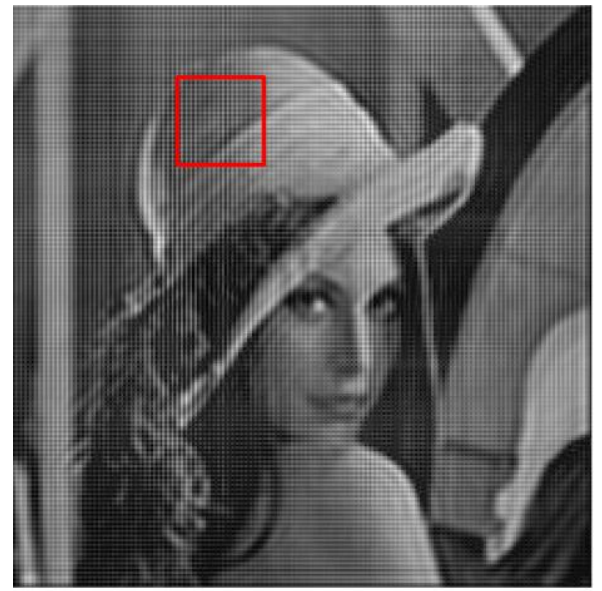

(a)

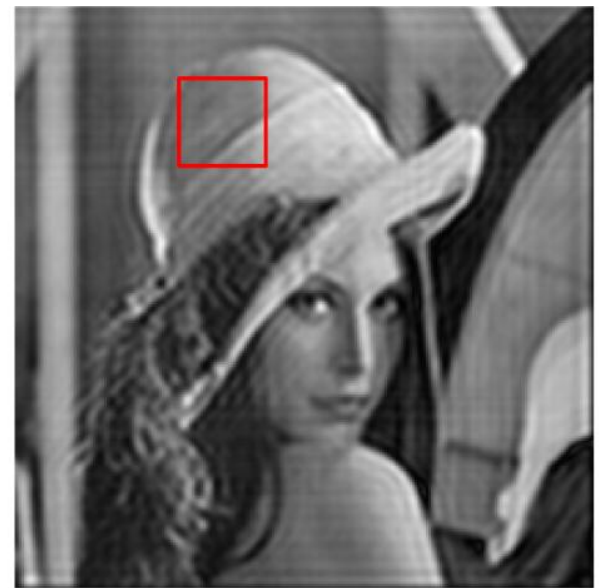

(b)

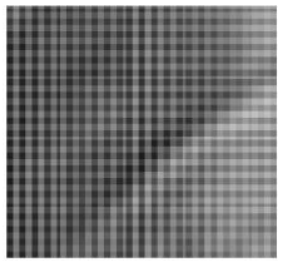

(c)

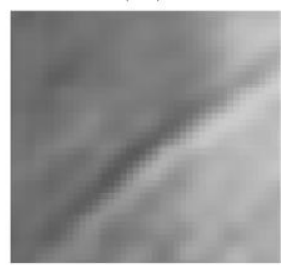

(d) 
Fig. 3. Simulated results of an extended image. Reconstructed results obtained using (a) propagation method and (b) CS algorithm., (c) and (d) shows the corresponding magnified areas from the (a) and (b).

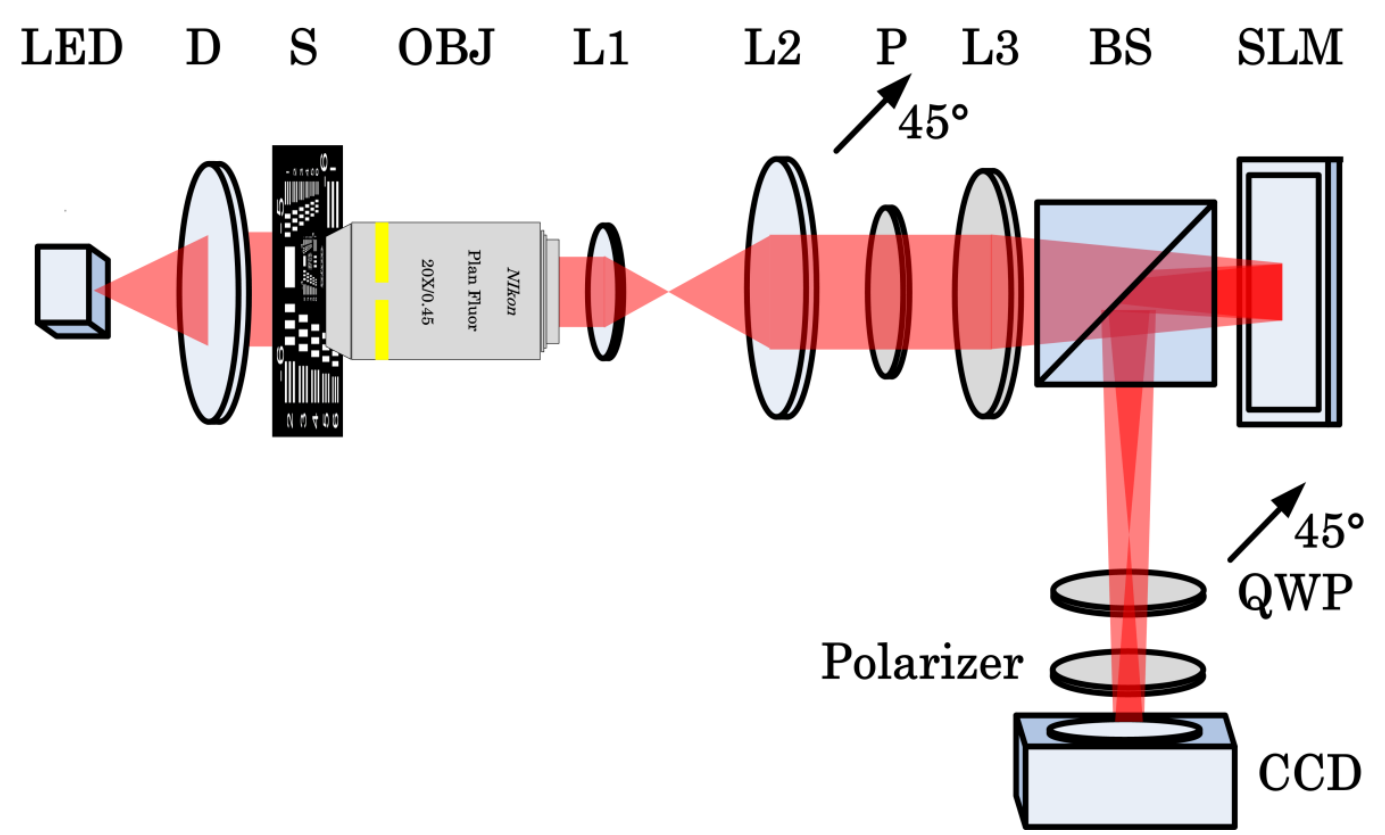

Fig. 4. Optical setup for PPSSIDH. The system consist of LED source; D, diffuser; S, sample; OBJ, microscope objective; L1-L3, lenses; P, polarizer; BS, beam splitter; SLM, spatial light modulator; CCD digital camera.

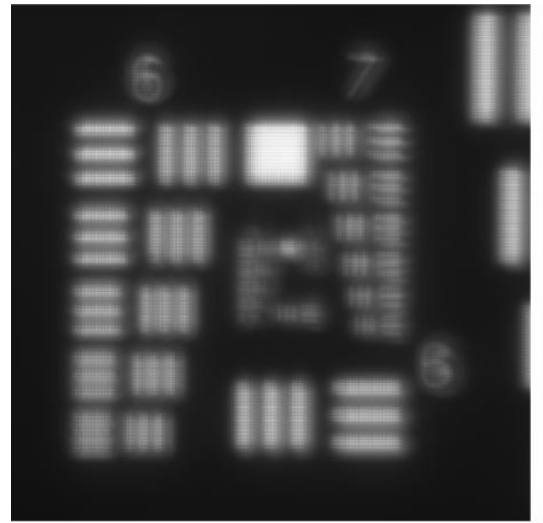

(a)

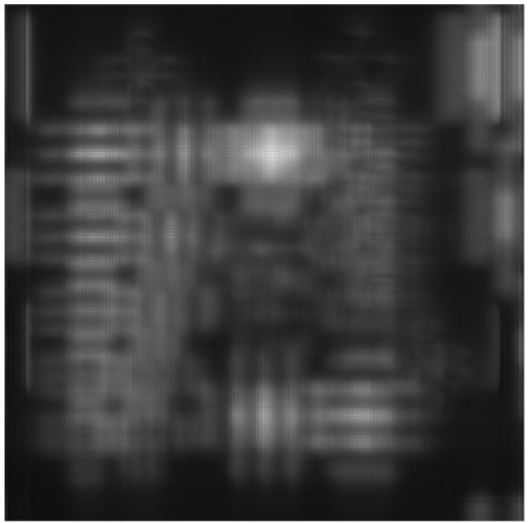

(b)

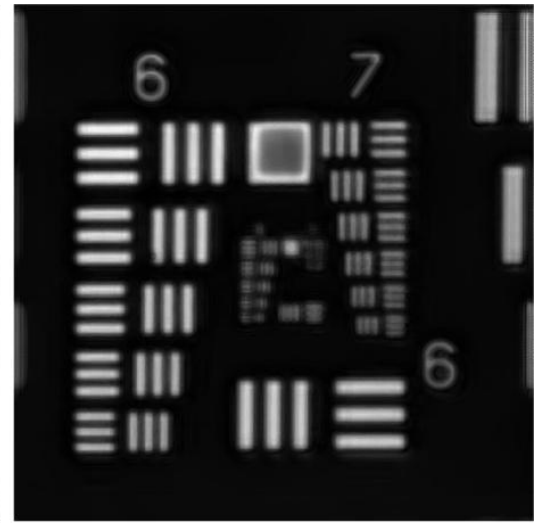

(c)

Fig. 5. Experimental results. (a) PPSSIDH hologram and reconstructions using (b) Fresnel propagation and (c) CS algorithms. 